\title{
Basics of Systematic Reviews and Meta-Analyses for the Nephrologist
}

\author{
Aminu K. Bello ${ }^{a}$ Natasha Wiebe ${ }^{a}$ Amit X. Garg ${ }^{b}$ Marcello Tonelli ${ }^{a}$ \\ a Division of Nephrology, University of Alberta, Edmonton, Alta., and ${ }^{b}$ Division of Nephrology, University of \\ Western Ontario, London, Ont., Canada
}

\section{Key Words}

Systematic review - Meta-analysis - Nephrologist •

Conduct $\cdot$ Appraisal $\cdot$ Reporting

\begin{abstract}
Renal practitioners are expected to apply the best available evidence from rigorous scientific research to clinical decision-making and also for policy-making for those involved. Advances in information technology and unprecedented access to data have simplified the process for the search of best available evidence to guide practice. However, it is challenging to cope with the increasing volume of publications in nephrology and other areas of medicine. Accordingly, systematic reviews and meta-analysis have greatly facilitated best practice and effective clinical decision-making. Conducting a systematic review/meta-analysis involves a number of steps that start with protocol development and research question formulation, design and study selection criteria, followed by retrieval of potentially relevant studies, selection of those studies to be included and evaluation of a study's risk of bias. Systematic reviews and meta-analyses have both strengths and weaknesses. Many of the perceived limitations of meta-analysis are not inherent in the methodology, but actually represent deficits in the conduct or reporting of individual primary studies. With the continuous proliferation of published renal clinical studies, such publica-
\end{abstract}

tions will continue to be an important resource for clinicians and researchers in nephrology. It is therefore important for nephrologists to keep abreast of developments in this field, which requires some knowledge about how these studies are conducted, reported and how to appraise them for application to clinical practice or policy-making.

Copyright $\odot 2011$ S. Karger AG, Basel

\section{Introduction}

Renal practitioners are expected to apply the best available evidence from rigorous scientific research to clinical decision-making [1-3]. Advances in information technology and unprecedented access to data have simplified the process for the search of best available evidence to guide practice $[1,2,4,5]$. However, it is challenging to cope with the increasing volume of publications in nephrology and other areas of medicine [6]. Accordingly, systematic reviews and meta-analysis have greatly facilitated best practice and effective clinical decision-making.

Systematic reviews and meta-analysis relevant to nephrologists might include evaluating the safety and efficacy of drug therapy (renal safety of tenofovir in the HIV-infected patients [7], erythropoiesis-stimulating agents in patients with chronic kidney disease [8], ascorbic acid for anaemia management in haemodialysis pa-

\section{KARGER}

Fax +4161306 1234 E-Mail karger@karger.ch www.karger.com
(C) 2011 S. Karger AG, Basel

$1660-2110 / 11 / 1191-0050 \$ 38.00 / 0$

Accessible online at:

www.karger.com/nec
Dr. Marcello Tonelli

University of Alberta, 7-129 Clinical Sciences Building

Edmonton, AB T6G 2G3 (Canada)

Tel. +1 7804078520

E-Mail mtonelli-admin@med.ualberta.ca 
tients [3], trace elements in haemodialysis patients [4], antibiotics for prophylaxis against haemodialysis catheterrelated infections [9], statins for improving renal outcomes [10]), outcomes due to applying an established treatment in a new population (cardiovascular effects of ACEi or ARBs in haemodialysis subjects [11]), the association between a risk parameter and outcome (link between eGFR and albuminuria with all-cause and CV mortality in the general population [12], chronic kidney disease and postoperative mortality [13] or all-cause mortality risk [14]) or testing the utility of certain procedures in clinical practice (ultrasound monitoring for access stenosis in haemodialysis patients [15]).

This article will review the basics of systematic literature review and meta-analysis.

\section{What Are Systematic Reviews and Meta-Analyses?}

A systematic review is an organized and structured evaluation of a clinical or research problem based on multiple independent studies that address the problem of interest [16-18]. Systematic review is the process of searching, selecting, appraising, synthesizing and reporting evidence from an existing body of published or unpublished literature. The articles to be reviewed are identified from a systematic and comprehensive search of the literature and are selected according to criteria set before beginning work. Data are then systematically abstracted from the reviewed articles, compiled into evidence tables, and then interpreted in the context of all relevant studies $[6,16,19]$. These steps distinguish systematic review from traditional narrative reviews, which often use subjective methods to collect and interpret studies, and are seldom explicit about study selection, assessment or analysis. This approach can lead to error and/or bias, making systematic reviews superior through their use of systematic methods: assessing and critically evaluating the literature in an organized, transparent and standard manner $[16$, $17,20,21]$.

\section{Why Use Systematic Reviews and Meta-Analyses?}

First, for busy healthcare providers and decision-makers, systematic reviews summarize the overwhelming amount of research-based healthcare evidence available for use in clinical decision-making and/or health policy formulation $[1,22,23]$. The aggregation of evidence, particularly if there is replication of results across studies, will often increase the certainty of the findings. Second, meta-analysis and systematic reviews overcome some of the biases and natural variation inherent in small studies where results may not be robust against chance variation - especially for small treatment effects. Similarly, systematic reviews overcome the lack of generalizability inherent in studies conducted in one particular type of population by including many trials conducted in varying populations. The differences in findings between studies can also be explored, which can yield new insights. These strengths of systematic reviews and meta-analysis stem from the rigorous design and conduct of the studies [22, $24,25]$.

Systematic reviews provide a systematic overview of study results on a certain subject by including all available information from similar individual studies that, viewed separately, may reach inconclusive results due to a relatively small sample size [26]. The term meta-analysis is often mistakenly used interchangeably with systematic review; meta-analysis is an optional component of a systematic review that statistically combines the data from individual studies. By combining those individual studies in a meta-analysis, the study power can be increased substantially, resulting in effect estimates that are more precise [26]. Initially, meta-analyses tended to include only randomized controlled trials (RCTs), but now increasingly include observational studies [27] to explore research questions that cannot be directly addressed in trials. The predefined and explicit methodology of a systematic review includes steps to minimize bias in all parts of the process: identifying relevant studies, selecting them for inclusion, and collecting and combining their data. The articles that result from this process can be helpful for clinical decisions, and they may also serve as the foundation for evidence-based practice guidelines, economic evaluations, and future research agendas [24, 28-30].

Physicians make better clinical decisions when they understand the circumstances and preferences of their patients, and combine their personal experience with clinical evidence underlying the available options. The public and professional organizations also expect that physicians will integrate research findings into their practice in a timely way [30]. Thus, sound clinical or health policy decisions are facilitated by reviewing the available evidence, understanding reasons why some studies differ in their results (a finding referred to as heterogeneity among the primary studies), coming up with an assessment of the expected effect of an intervention or exposure, and then integrating the new information with 
other relevant treatment, patient and health care system factors.

Thus, reading a properly conducted systematic review is an efficient way of becoming familiar with the best available research evidence. If the review team has obtained unpublished information from the authors of primary studies, a systematic review can also extend the available literature. Reviewing the evidence summary allows the reader to establish whether the scientific findings are consistent and valid across populations, settings and treatment variations, and whether findings vary significantly by particular subgroups.

\section{How Are Systematic Reviews Conducted?}

A series of guidelines has been published over the last decade to describe how to report studies on therapy [31], screening or diagnostic [32], cost-effectiveness [33], or prognostic issues [34]. In 1996, the Quality of Reporting of Meta-Analyses (QUORUM) statement was published to improve specifically the quality of reporting metaanalyses of RCTs [35]. In the year 2000, there was the publication of Meta-analysis Of Observational Studies in Epidemiology (MOOSE) guidelines for reporting systematic reviews of observational studies [21]. These statements include a checklist which describes the preferred way to report a systematic review/meta-analysis. Recently, these guidelines have been updated by the publication of PRISMA (Preferred Reporting Items for Systematic reviews and Meta-Analyses), which have been updated to address several conceptual and practical advances in the science of systematic reviews [36]. Several recent articles describe how to conduct a systematic review $[16,24,25$, $28,37-39]$. Conducting a systematic review/meta-analysis involves a number of steps that start with protocol development and research question formulation, design and study selection criteria, followed by retrieval of potentially relevant studies, selection of those studies to be included and evaluation of a study's risk of bias (table 1). Thereafter, if justifiable, an actual meta-analysis may be performed, reasons why the results of primary studies differed will be considered (qualitative and quantitative heterogeneity), and finally sensitivity analyses are performed to ensure that bias did not influence the result $[40$, 41].

As in all types of research, development of a well-written protocol is paramount to define standard procedures (research questions, appropriate methodology, data retrieval process and analysis plan). This will minimize
Table 1. Steps to undertake a systematic review

Defining a research question: The problems to be addressed by the review should be identified, with the objectives of the review clearly stated. A prospective protocol defines the populations, inclusion/exclusion criteria, interventions, study designs and outcomes.

Literature search: The published and unpublished literature should be carefully searched for relevant studies required to answer the research question of interest. A professional librarian should help to design the search, where possible.

Study selection: Once all possible records of studies have been identified, they should be independently screened by two reviewers for potential eligibility. The full-text papers would then be retrieved whereby each potential study would be thoroughly assessed against a prior inclusion and exclusion criteria by two independent reviewers.

Data abstraction: Of the included studies, relevant characteristics and results should be abstracted onto a data abstraction form or database. Some studies will be excluded even at this late stage. Primary articles should be processed for risk of bias (using an evidence-based framework).

Synthesis, exploration for heterogeneity, and reporting of the results: The findings from the individual studies should be synthesized, aggregated and reported - all according to the initially proposed protocol. Deviations to or addition from the protocol should be clearly noted and mentioned in the report.

Placing the findings in context: The findings from the evidence synthesis should then be put into the context of the existing literature. This will address issues such as the quality and heterogeneity of the included studies, the likely impact of bias as well as the applicability of the findings to practitioners.

bias and increase the chance that the conclusions of the review are valid $[26,37,42]$. The primary step is to formulate a focused and clearly defined research question $[16,42,43]$. It is always better to frame a narrow and specific question. A broad question such as 'what is the most appropriate prophylactic measure for the prevention of haemodialysis catheter infections' would be better framed as 'what is the role of antibiotics or the role of catheter lock solutions in the prevention of access-related infections in haemodialysis patients'. It is recommended to describe the primary question in terms of the 'PICO' framework (Population, Intervention/exposure, Control/comparator and Outcomes of interest) $[43,44]$.

After formulating the research question and writing the protocol, the next stages are to perform a comprehensive and systematic literature search, to select potentially relevant articles, and to abstract data from primary stud- 
ies. Peer-reviewed biomedical journals are the most common source of information, and electronic bibliographic databases are used to search for relevant articles. It is important to seek the assistance of a librarian educated in conduct of systematic reviews. The predominant databases in use are MEDLINE and EMBASE. There are some key differences between EMBASE and MEDLINE, and in most cases the review team should have searched both. For example, EMBASE provides the best coverage of European research as well as pharmaceutical research including renal adverse events [45]. On the other hand, positive studies are more often published in journals which are indexed in MEDLINE, compared to non-indexed journals [46]. 'Grey' literature too finds less positive results than formally published literature [47]. As a supplementary method of identifying information, searching databases such as the Cochrane Library (which identifies many papers through manual hand-searching of journals and conference proceedings), the Science Citation Index (which identifies papers which cite the relevant articles) as well as newer internet search engines like Google Scholar and Elsevier's Scirus can be useful for identifying articles not indexed well in traditional bibliographic databases. Searching bibliographies of previous systematic reviews and primary articles can also identify relevant articles that were missed. Whatever bibliographic sources were used, the review team should have employed a search strategy that maximized the identification of relevant articles.

The next three steps should be conducted in duplicate. First, two independent reviewers should evaluate each potentially relevant abstract for inclusion using a predrafted form stipulating the PICO criteria plus essential study characteristics (such as type of design, length of follow-up, minimum sample size). Second, articles deemed relevant by at least one reviewer on review of the abstract should be retrieved, and the full text reviewed in duplicate, again using a pre-drafted form. Disagreements about which articles are eligible for inclusion should be resolved through discussion or with the aid of a third party. Third, relevant data should be abstracted and risk of bias components should be assessed - both in duplicate. The term 'risk of bias' has replaced the previously used term 'quality assessment' $[48,49]$ since minimizing bias is more relevant to studies than the more abstract objective of ensuring 'quality'. For example, although blinded studies are felt to be of higher 'quality' than unblinded studies - trials in which the primary outcome is mortality may not have appreciable risk of bias even if those who assess outcomes are aware of treatment assignment.
The next stage is to pool the included studies. The more similar the studies and their results, the more reasonable it is to pool them together for analysis $[43,50]$. The decision to combine studies should be based on both qualitative and quantitative evaluation. These involve assessing the homogeneity of populations, interventions used, key objectives, outcome definition (including the individual components of a composite outcome) and duration of follow-up among the underlying studies. If the studies are sufficiently homogeneous that pooling their results is reasonable, meta-analysis can be done. Basic meta-analysis combines results across studies to provide overall estimates and confidence intervals of treatment effects [39]. For meta-analysis for a binary outcome, the odds ratio or relative risk, which measure the relative effect, in general are preferable to the metrics that measure absolute differences, such as risk difference because relative measures are more likely to be robust to variations in baseline risk [51].

Data from individual studies are not combined using simple arithmetic. Rather, greater weights are given to the results from studies that provide more information (larger sample size and smaller variance), because statistically they are likely to be closer to the true effect being estimated (assuming the risk of bias with respect to study methods is similar across the studies). Quantitatively, summary effect estimates will be calculated under the assumption that the intervention or exposure has a 'fixed' or 'random' effect $[39,50]$ - meaning that the true estimate of effect is either static or has itself some random variation. The assumption of random effects is more relaxed and expects small levels of heterogeneity in population, intervention/exposure and design.

It is also useful to evaluate and explore heterogeneity between studies [20]. The magnitude of heterogeneity may be quantified with a statistic referred to as the $\mathrm{I}^{2}$, which describes the percentage of variability due to between-study differences as opposed to between-participant differences. Values of $0-25,26-50$, and $>50 \%$ represent mild, moderate, and notable heterogeneity, respectively [52]. When there is substantial heterogeneity, it may be more appropriate to report the individual results without pooling, using a forest plot. To explore substantial heterogeneity, a review may report pooled estimates in important subgroups. Additionally, meta-regression may be used where each primary study estimate is regressed on study-level (or group-level) estimates of potential modifiers [53]. Both these techniques are limited by ecological fallacy (meaning that a relationship between predictor and outcome may appear one way when the unit of 
Table 2. Assessing the methodological quality of a systematic review

(1) A clear focused question with an a priori protocol and transparent methodology. Was the choice of study design and comparator group appropriate for the question?

(2) A systematic and extensive literature search $[70,71]$. An experienced librarian would be an asset.

(3) Study selection, data abstraction and risk of bias assessment performed in duplicate.

(4) Risk of bias included items with empirical evidence: for example, the method to generate the randomization list [58], concealment of therapy assignment (prior to assignment) [72], blinding of study participants and outcome assessors [58], completeness of follow-up regardless of protocol adherence [58], selective outcome reporting [73], stopping early [74], source of funding [75].

(5) Heterogeneity (and incoherence in network meta-analysis) assessed and explored if present. An appropriate subject-specialist and experienced statistician recommended.

(6) Publication (also known as small study) bias considered [76, 77].

analysis is a patient within a study, but completely different when observations are made across studies and the unit of analysis is the study itself).

A new technique that facilitates comparisons of multiple therapies is network meta-analysis [54, 55]. In addition to direct evidence (evidence from studies that directly compare two or more therapies), indirect evidence is incorporated into the overall pooled result. In its simplest form, the estimates from studies that compare therapy $\mathrm{A}$ to therapy $\mathrm{C}$, and studies that compare therapy $\mathrm{B}$ to therapy $\mathrm{C}$ can be subtracted (thereby removing the effect of therapy C) - resulting in an indirect estimate of therapy A compared to therapy B [56]. If the trials studying therapy are too different from one another, then the resulting network would be incoherent (also known as inconsistent) [57] and should not be recommended as reliable evidence.

The last step is interpreting reporting the results whether the results have been combined statistically or not. The new PRISMA report is now the standard framework for reporting systematic reviews and meta-analyses of both RCTs and observational studies, [36] and has adopted the definitions used by the Cochrane Collaboration.

\section{Interpretation and Application of a Systematic Review and Meta-Analysis}

Like all research techniques, meta-analysis can be done with and without appropriate methodology. Before considering the results, there are a few questions that readers can ask themselves when assessing the method- ological quality of a systematic review. First, the date of the review's publication should be considered in light of the rate at which new studies are being published. Keeping abreast of new developments is paramount as quite often new data are published that can influence the results and interpretation of the previously published review.

Tools for assessing risk of bias or the methodological quality of a systematic review are not widely in use. We present six items that can be used to assess methodological rigor (table 2). Some items are supported with empirical validation and others have only face validity.

Other items that have been investigated have little supportive evidence: obscuring out author names and affiliations [58-60], non-English language publications [61, 62], peer-review manuscripts [63], hard to locate ('grey') literature $[64,65]$, independent double data abstraction [66], and experienced data abstractors [67].

\section{Interpretation of a Systematic Review}

Ideally three types of figures are included with a systematic review: a flow diagram of study selection (fig. 1) $[25,43]$, a forest plot depicting individual and most often an overall pooled estimate of effect (fig. 2) [26, 43, 68], and a funnel plot showing an assessment of publication bias (fig. 3).

Systematic reviews can be summarized using tables. However, meta-analyses are typically graphed using a forest plot. A forest plot contains the individual study point estimates and their associated $95 \%$ confidence intervals (CI). Confidence limits that contain 1 if the measure of effect is relative (or 0 if the measure of effect is absolute), indicate a non-statistically significant differ- 
Fig. 1. Example of a PRISMA flow chart. X represents the number of studies in each category. Reasons for exclusion are examples only.
Potentially relevant records identified through database searching $(n=X)$ and through other sources $(n=X)$

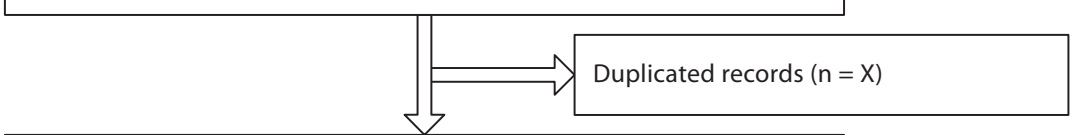

Potentially relevant records identified and screened for retrieval $(n=X)$

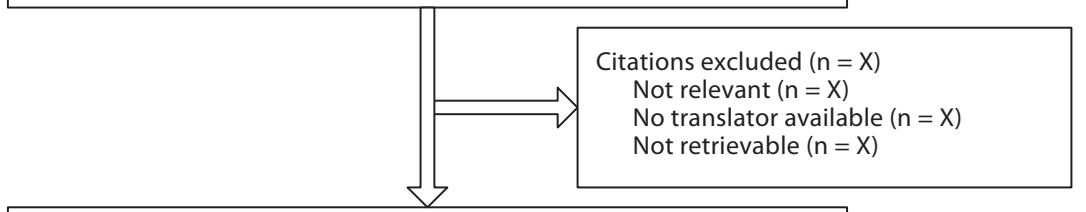

Articles retrieved for detailed evaluation $(n=X)$

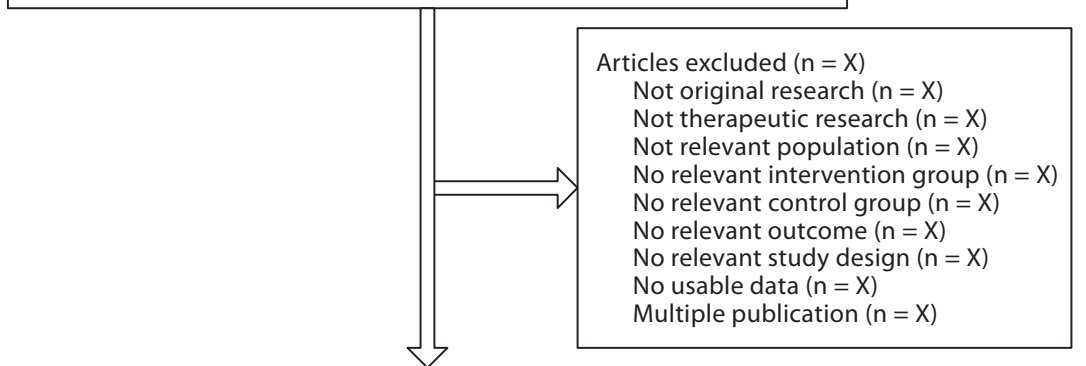

Primary articles included in the systematic review $(n=X)$ ence in effect. Confidence limits may also include differences too small to be clinically important [69] and should be deemed as neither evidence of an 'important effect' nor evidence of 'no difference' in effect. The forest plot also allows one to appreciate the heterogeneity of results, by comparing the deviances of the individual studies from each other and from the overall result.

A funnel plot is a simple scatter plot of each study's precision (inversion of standard error) on the $y$-axis against each study's effect on the x-axis. Because small studies have less precision and large studies have more, scatter should form an inverted funnel when there are no systematic missing studies. A line is often drawn through the overall pooled effect to aid the eye in detecting symmetry (an inverted funnel) or asymmetry. Asymmetry suggests missing evidence, which often represents small, negative studies.

The meta-regression plot is not widely used (fig. 4). Because the unit of analysis in this form of regression is the study rather than the participant or patient, the analysis is typically underpowered and statistical significance is rare. The meta-regression plot is both a scatter and line plot. Each study's estimate of effect is plotted against the value of the potential modifier and a regression line is drawn through the scatter of observations. A slope indicates the direction and whether there is an association between the potential modifier and the effect estimate.

In publications of network meta-analyses, due to multiple intervention groups and the complexity therein, a figure depicting the number of comparisons between each set of interventions is usually provided (fig. 5). Often a matrix of direct and mixed evidence for each comparison is reported, rather than a forest plot showing the pooled and each individual study's estimate. The matrix, a square table with the intervention labels running diagonally along the centre, allows one to appreciate what direct evidence is absent and where 
Fig. 2. Example of a forest plot. Each row of a forest plot (also called a meta-graph) represents information pulled from one study or the total or a subgroup of pooled studies. The marker represents the point estimate of effect. The width of the bar represents the 95\% CI. A diamond marker usually indicates the total or a subgroup total of pooled results. In this example, Y is the point estimate of effect for each trial, and $\mathrm{X}, \mathrm{Z}$ are the $95 \% \mathrm{CI}$. To the left of the plot is tabular information for each study, often the sample size of each group or study and the numerical point and interval estimates.

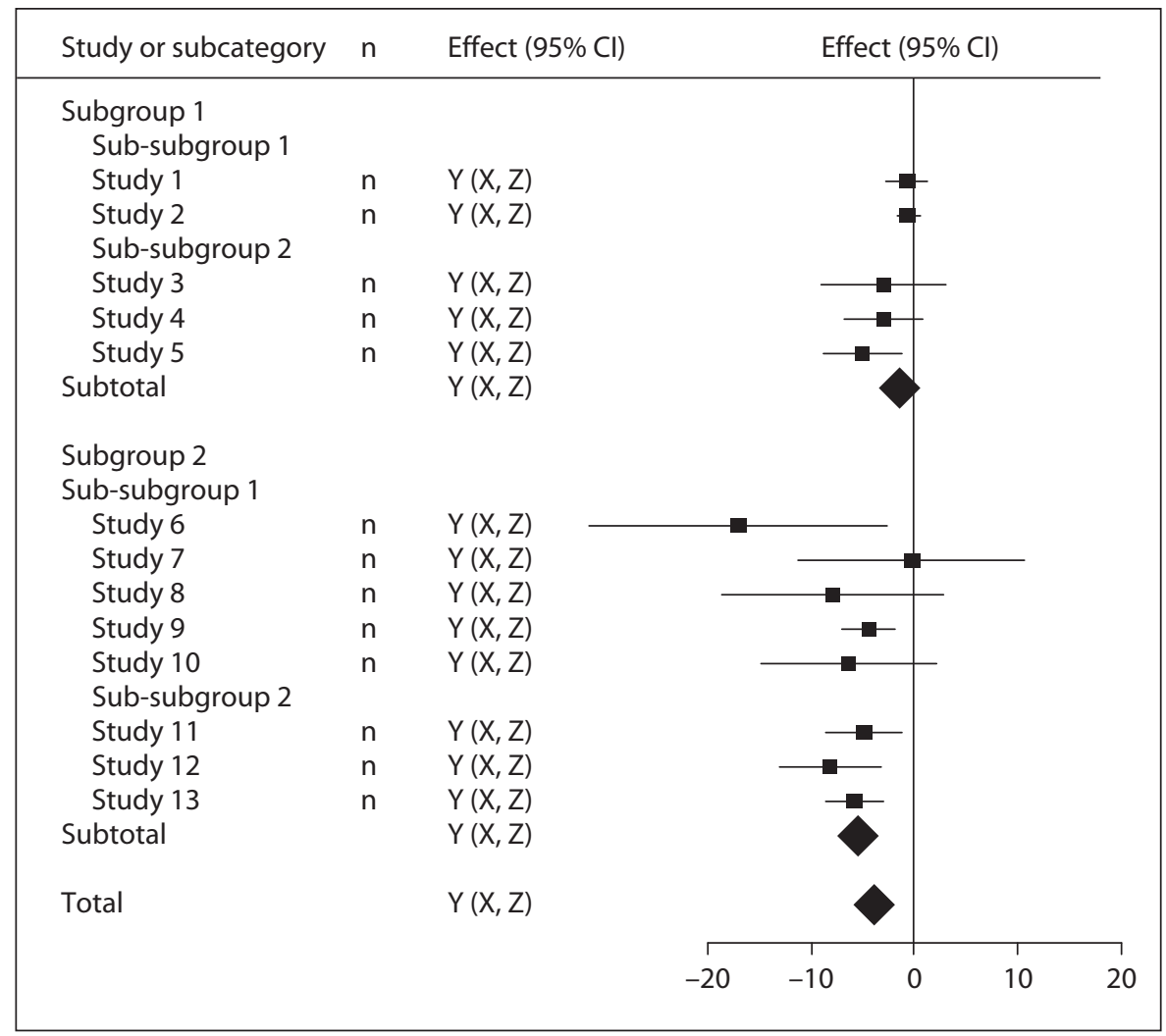

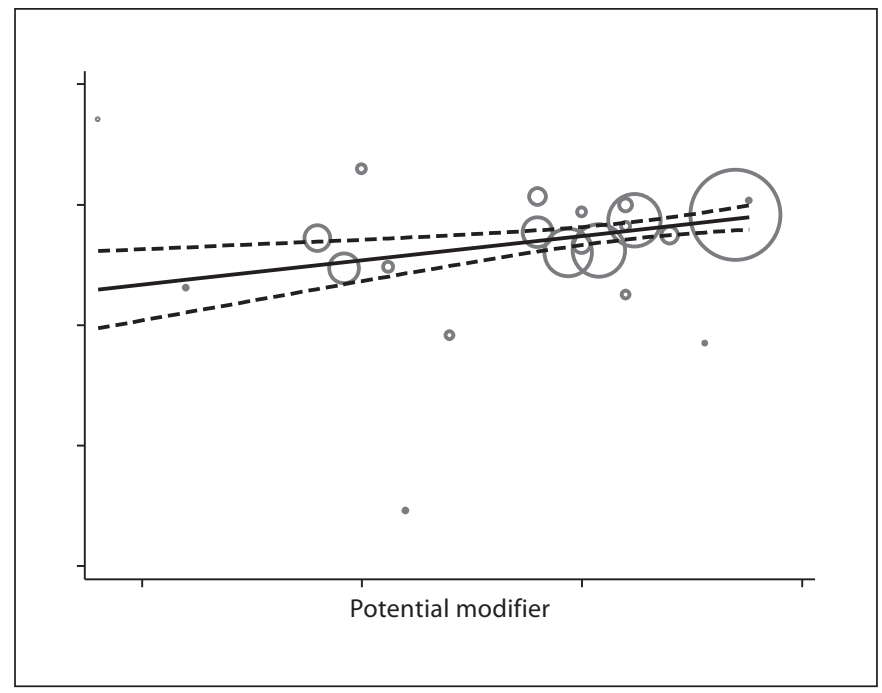

Fig. 3. Example of a funnel plot. Each study's precision (the inverse of the standard error of each study's effect estimate) is plotted against each study's effect estimate. These markers may be sized according to the study's sample size; larger studies are marked with larger circles. A vertical line is drawn through our overall pooled estimate of effect to aid the eye in detecting symmetry (an inverted funnel) or asymmetry. This funnel plot appears mildly asymmetric. The emptier right side of the inverted funnel may indicate small, missing studies.
Fig. 4. Example of a meta-regression plot. Each primary study's estimate of effect is plotted against a variable that may potentially modify the relationship between outcome and intervention (or exposure). The markers (circles) are sized according to precision - the inverse of the standard error of each study's effect estimate. The three lines are the fitted (solid) and the upper and lower bounds (dashed) of the $95 \%$ CI. 
Fig. 5. Example of a network meta-analysis figure. Each circle represents an intervention group and each line drawn between each set of intervention groups shows the number $\mathrm{X}$ of studies that directly compared these respective interventions. No line means that no study compared the respective interventions directly.

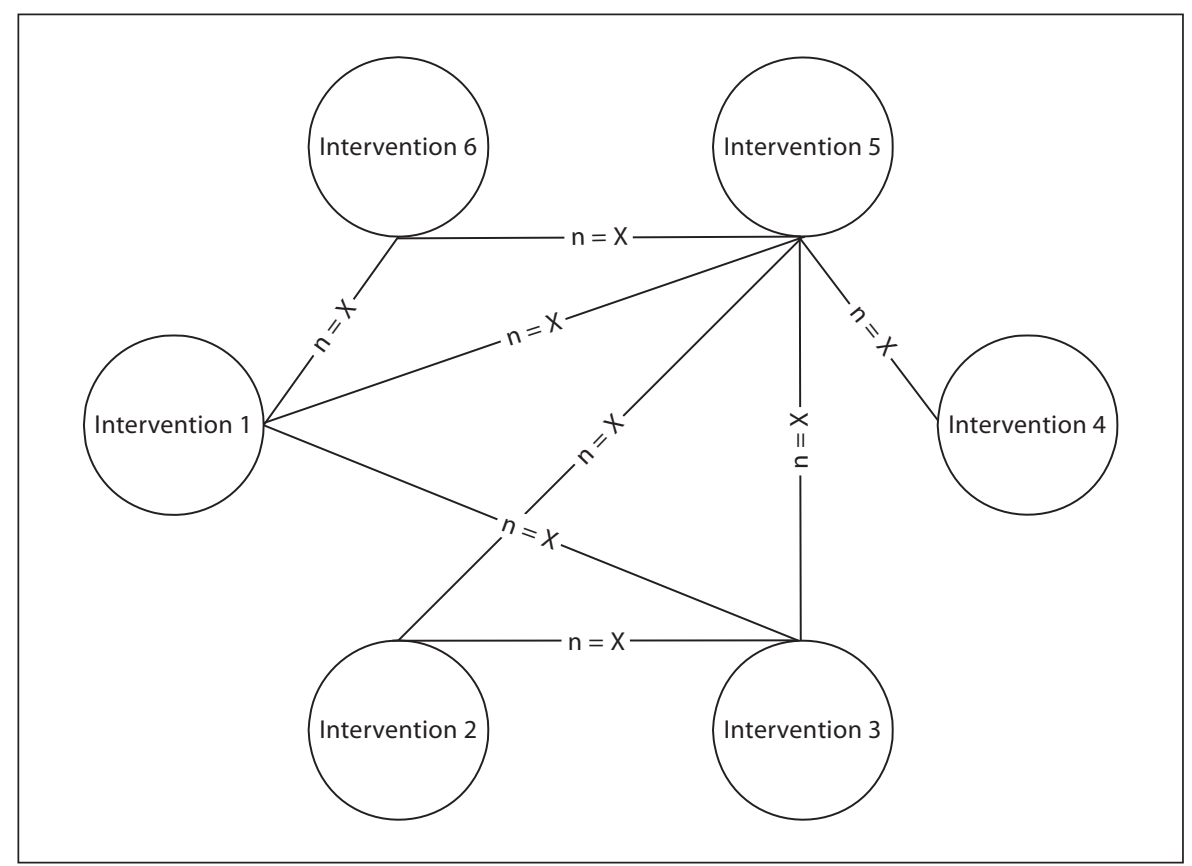

\begin{tabular}{|l|l|l|l|l|l|}
\hline Intervention 6 & $\mathrm{Y}(\mathrm{X}, \mathrm{Z})$ & $\mathrm{Y}(\mathrm{X}, \mathrm{Z})$ & $\mathrm{Y}(\mathrm{X}, \mathrm{Z})$ & $\mathrm{Y}(\mathrm{X}, \mathrm{Z})$ & $\mathrm{Y}(\mathrm{X}, \mathrm{Z})$ \\
\hline $\mathrm{Y}(\mathrm{X}, \mathrm{Z})$ & Intervention 5 & $\mathrm{Y}(\mathrm{X}, \mathrm{Z})$ & $\mathrm{Y}(\mathrm{X}, \mathrm{Z})$ & $\mathrm{Y}(\mathrm{X}, \mathrm{Z})$ & $\mathrm{Y}(\mathrm{X}, \mathrm{Z})$ \\
\hline- & $\mathrm{Y}(\mathrm{X}, \mathrm{Z})$ & Intervention 4 & $\mathrm{Y}(\mathrm{X}, \mathrm{Z})$ & $\mathrm{Y}(\mathrm{X}, \mathrm{Z})$ & $\mathrm{Y}(\mathrm{X}, \mathrm{Z})$ \\
\hline- & $\mathrm{Y}(\mathrm{X}, \mathrm{Z})$ & - & Intervention 3 & $\mathrm{Y}(\mathrm{X}, \mathrm{Z})$ & $\mathrm{Y}(\mathrm{X}, \mathrm{Z})$ \\
\hline- & $\mathrm{Y}(\mathrm{X}, \mathrm{Z})$ & - & $\mathrm{Y}(\mathrm{X}, \mathrm{Z})$ & Intervention 2 & $\mathrm{Y}(\mathrm{X}, \mathrm{Z})$ \\
\hline $\mathrm{Y}(\mathrm{X}, \mathrm{Z})$ & $\mathrm{Y}(\mathrm{X}, \mathrm{Z})$ & - & $\mathrm{Y}(\mathrm{X}, \mathrm{Z})$ & - & Intervention 1 \\
\hline
\end{tabular}

Fig. 6. Example of a network meta-analysis matrix. The mixed evidence appears in the shaded upper triangle and the corresponding direct evidence appears in the unshaded lower triangle. A dash indicates no available direct evidence (meaning that trials specifically comparing this pair of treatments were not identified in the review). In this example, $\mathrm{Y}$ is the point estimate of effect for each trial, and $\mathrm{X}, \mathrm{Z}$ are the $95 \% \mathrm{CI}$.

the mixed evidence does not agree with the direct evidence (fig. 6). The row-column cell of the available direct evidence corresponds to the column-row cell of the mixed evidence.

A good understanding of common statistical terms used in meta-analyses is also important for interpretation and application of systematic reviews and meta-analysis (table 3).

\section{Application of a Systematic Review}

The application of systematic review/meta-analysis in clinical practice or policy-making requires careful con- sideration and appraisal. One needs to start with the research question at hand, and then decide whether a systematic review is a sensible approach to answer that particular question $[25,36]$. If so, has the systematic review adequately addressed the question [36]? If the study has been adequately performed and the risk of bias is judged acceptably low, the next step will be to decide on the findings' applicability to the reader's setting, population or patient group. Factors that should be considered in this assessment include the baseline characteristics of the study subjects, follow-up, and study design and detailed inclusion/exclusion criteria. The results (absolute benefit, 
Table 3. Common statistical terms used in meta-analyses

\begin{tabular}{|c|c|c|}
\hline Measure & Description & $\begin{array}{l}\text { Example } \\
\text { For all examples assume that the value of each } \\
\text { measure }=2\end{array}$ \\
\hline \multicolumn{3}{|l|}{ For pooling } \\
\hline $\begin{array}{l}\text { Relative risk or } \\
\text { risk ratio (RR) }\end{array}$ & $\begin{array}{l}\text { Risk of the experimental group divided by the } \\
\text { risk of the control group. Risk is another term } \\
\text { for probability. }\end{array}$ & $\begin{array}{l}\text { Participants with the experimental therapy had } \\
2 \text { times the risk of experiencing the outcome } \\
\text { than participants with the control therapy. }\end{array}$ \\
\hline Odds ratio (OR) & $\begin{array}{l}\text { Odds of the experimental group divided by the } \\
\text { odds of the control group. The OR will appear to } \\
\text { exaggerate the estimate of RR. }\end{array}$ & $\begin{array}{l}\text { Participants with the experimental therapy had } \\
2 \text { times the odds of experiencing the outcome } \\
\text { than participants with the control therapy. }\end{array}$ \\
\hline Risk difference (RD) & $\begin{array}{l}\text { Risk of the experimental group minus the risk of } \\
\text { the control group. }\end{array}$ & $\begin{array}{l}\text { Participants with the experimental therapy had } \\
2 \% \text { more risk of experiencing the outcome than } \\
\text { participants with the control therapy. }\end{array}$ \\
\hline $\begin{array}{l}\text { Number needed to } \\
\text { treat (NNT) }\end{array}$ & $\begin{array}{l}\text { The NNT is not used to pool results from } \\
\text { individual studies because its variance is difficult } \\
\text { to estimate. It may be calculated from RD, RR, } \\
\text { or OR along with an estimate of control risk to } \\
\text { clarify the degree of effect. }\end{array}$ & $\begin{array}{l}\text { In order to prevent one poor outcome, two } \\
\text { participants need to be treated with the } \\
\text { experimental therapy rather than with the } \\
\text { control therapy. }\end{array}$ \\
\hline Mean difference (MD) & $\begin{array}{l}\text { Mean of the experimental group minus the } \\
\text { mean of the control group. It is a difference of } \\
\text { means rather than a mean of differences. }\end{array}$ & $\begin{array}{l}\text { Participants with the experimental therapy had } \\
2 \text { units more, on average, of the outcome than } \\
\text { participants with the control therapy. }\end{array}$ \\
\hline \multicolumn{3}{|l|}{ For exploring heterogeneity } \\
\hline $\mathrm{I}^{2}$ & $\begin{array}{l}\text { The percent of variance due to between-study } \\
\text { variance as opposed to between-participant (or } \\
\text { within-study) variance. }\end{array}$ & $\begin{array}{l}2 \% \text { of the total variance is due to between- } \\
\text { study differences. Since this value is much } \\
\text { smaller than } 25 \% \text {, there is very little between- } \\
\text { study heterogeneity in this effect estimate. }\end{array}$ \\
\hline $\begin{array}{l}\text { Relative risk ratio } \\
\text { (RRR) }\end{array}$ & $\begin{array}{l}\text { The relative risk of studies with the potential } \\
\text { modifier divided by the relative risk of the } \\
\text { studies without the potential modifier. }\end{array}$ & $\begin{array}{l}\text { Studies with the potential modifier had a } \\
\text { relative risk of experiencing the outcome } 2 \\
\text { times as large as studies without the potential } \\
\text { modifier. }\end{array}$ \\
\hline $\begin{array}{l}\text { Difference of mean } \\
\text { difference (MDD) }\end{array}$ & $\begin{array}{l}\text { The mean difference of studies with the } \\
\text { potential modifier minus the mean difference of } \\
\text { the studies without the potential modifier. }\end{array}$ & $\begin{array}{l}\text { Studies with the potential modifier had a mean } \\
\text { difference that was } 2 \text { units greater than studies } \\
\text { without the potential modifier. }\end{array}$ \\
\hline
\end{tabular}

effectiveness and harm) should all be considered from the point of view of the individual and the population at large. Finally, the reader must decide on whether policy or clinical practice should change as a result of the evidence summary [36].

\section{Conclusion}

Systematic reviews and meta-analyses have both strengths and weaknesses. Many of the perceived limitations of meta-analysis are not inherent in the method- ology, but actually represent deficits in the conduct or reporting of individual primary studies. With the continuous proliferation of published renal clinical studies, such publications will continue to be an important resource for clinicians and researchers in nephrology. It is therefore important for them to keep abreast of developments in this field, which requires some knowledge about how these studies are conducted and how to appraise them. 


\section{References}

1 Campbell MK, Daly C, Wallace SA, Cody DJ, Donaldson C, Grant AM, et al: Evidencebased medicine in nephrology: identifying and critically appraising the literature. Nephrol Dial Transplant 2000;15:19501955.

-2 Blettner M, Sauerbrei W: Meta-analysis of epidemiologic studies (in German). Med Klin (Munich) 1998;93:442-445.

- 3 Deved V, Poyah P, James MT, Tonelli M, Manns BJ, Walsh M, et al: Ascorbic acid for anemia management in hemodialysis patients: a systematic review and meta-analysis. Am J Kidney Dis 2009;54:1089-1097.

-4 Tonelli M, Wiebe N, Hemmelgarn B, Klarenbach S, Field C, Manns B, et al: Trace elements in hemodialysis patients: a systematic review and meta-analysis. BMC Med 2009;7: 25.

5 Sackett DL, Rosenberg WM: The need for evidence-based medicine. J R Soc Med 1995; 88:620-624.

-6 Noordzij M, Hooft L, Dekker FW, Zoccali C, Jager KJ: Systematic reviews and meta-analyses: when they are useful and when to be careful. Kidney Int 2009;76:1130-1136.

$\checkmark 7$ Cooper RD, Wiebe N, Smith N, Keiser P, Naicker S, Tonelli M: Systematic review and meta-analysis: renal safety of tenofovir disoproxil fumarate in HIV-infected patients. Clin Infect Dis 2010;51:496-505.

8 Tonelli M, Klarenbach S, Wiebe N, Shrive F, Hemmelgarn B, Manns B: Erythropoiesisstimulating agents for anemia of chronic kidney disease: systematic review and economic evaluation. Ottawa, Canadian Agency for Drugs and Technologies in Health, 2008.

\ James MT, Conley J, Tonelli M, Manns BJ, MacRae J, Hemmelgarn BR: Meta-analysis: antibiotics for prophylaxis against hemodialysis catheter-related infections. Ann Intern Med 2008;148:596-605.

10 Sandhu S, Wiebe N, Fried LF, Tonelli M: Statins for improving renal outcomes: a meta-analysis. J Am Soc Nephrol 2006; 17:20062016.

-11 Tai DJ, Lim TW, James MT, Manns BJ, Tonelli M, Hemmelgarn BR: Cardiovascular effects of angiotensin-converting enzyme inhibition or angiotensin receptor blockade in hemodialysis: a meta-analysis. Clin J Am Soc Nephrol 2010;5:623-630.

-12 Matsushita K, van der Velde M, Astor BC, Woodward M, Levey AS, de Jong PE, et al: Association of estimated glomerular filtration rate and albuminuria with all-cause and cardiovascular mortality in general population cohorts: a collaborative meta-analysis. Lancet 2010;375:2073-2081.

13 Mathew A, Devereaux PJ, O’Hare A, Tonelli $\mathrm{M}$, Thiessen-Philbrook $\mathrm{H}$, Nevis IF, et al: Chronic kidney disease and postoperative mortality: a systematic review and metaanalysis. Kidney Int 2008;73:1069-1081.
4 Tonelli M, Wiebe N, Culleton B, House A, Rabbat C, Fok M, et al: Chronic kidney disease and mortality risk: a systematic review. J Am Soc Nephrol 2006;17:2034-2047.

15 Tonelli M, James M, Wiebe N, Jindal K, Hemmelgarn B: Ultrasound monitoring to detect access stenosis in hemodialysis patients: a systematic review. Am J Kidney Dis 2008;51:630-640.

16 Tonelli M, Hackam D, Garg AX: Primer on systematic review and meta-analysis. Methods Mol Biol 2009;473:217-233.

17 Yusuf S, Pogue J: Meta-analysis. Lancet 1998; 351:1960.

18 Sterne JA, Egger M, Smith GD: Systematic reviews in health care: investigating and dealing with publication and other biases in meta-analysis. BMJ 2001;323:101-105.

19 Simmonds MC, Higgins JP, Stewart LA, Tierney JF, Clarke MJ, Thompson SG: Metaanalysis of individual patient data from randomized trials: a review of methods used in practice. Clin Trials 2005;2:209-217.

20 Thompson SG: Why sources of heterogeneity in meta-analysis should be investigated. BMJ 1994:309:1351-1355.

21 Stroup DF, Berlin JA, Morton SC, Olkin I, Williamson GD, Rennie D, et al: Meta-analysis of observational studies in epidemiology: a proposal for reporting. Meta-analysis Of Observational Studies in Epidemiology (MOOSE) group. JAMA 2000;283:20082012.

22 Balk EM, Lau J, Bonis PA: Reading and critically appraising systematic reviews and meta-analyses: a short primer with a focus on hepatology. J Hepatol 2005;43:729-736.

23 Cook DJ, Greengold NL, Ellrodt AG, Weingarten SR: The relation between systematic reviews and practice guidelines. Ann Intern Med 1997;127:210-216.

24 Cook DJ, Sackett DL, Spitzer WO: Methodologic guidelines for systematic reviews of randomized control trials in health care from the Potsdam Consultation on MetaAnalysis. J Clin Epidemiol 1995;48:167-171.

25 Garg AX, Hackam D, Tonelli M: Systematic review and meta-analysis: when one study is just not enough. Clin J Am Soc Nephrol 2008:3:253-260

26 Jadad AR, Cook DJ, Jones A, Klassen TP, Tugwell P, Moher M, et al: Methodology and reports of systematic reviews and meta-analyses: a comparison of Cochrane reviews with articles published in paper-based journals JAMA 1998;280:278-280.

27 Claridge JA, Fabian TC: History and development of evidence-based medicine. World J Surg 2005;29:547-553.

28 Fouque D: Producing systematic reviews of best quality: a prerequisite for evidencebased nephrology. J Nephrol 1999;12:314317.
29 Flather MD, Farkouh ME, Pogue JM, Yusuf S: Strengths and limitations of meta-analysis: larger studies may be more reliable. Control Clin Trials 1997;18:568-579; discussion 661-666.

30 Egger M, Smith GD, Sterne JA: Uses and abuses of meta-analysis. Clin Med 2001;1: 478-484.

31 Moher D, Schulz KF, Altman DG: The CONSORT statement: revised recommendations for improving the quality of reports of parallel group randomized trials. BMC Med Res Methodol 2001;1:2.

-32 Bossuyt PM, Reitsma JB, Bruns DE, Gatsonis CA, Glasziou PP, Irwig LM, et al: The STARD statement for reporting studies of diagnostic accuracy: explanation and elaboration. Ann Intern Med 2003;138:W1-W12.

33 Drummond MF, Jefferson TO: Guidelines for authors and peer reviewers of economic submissions to the BMJ. The BMJ Economic Evaluation Working Party. BMJ 1996;313: 275-283.

34 Von Elm E, Altman DG, Egger M, Pocock SJ, Gotzsche PC, Vandenbroucke JP: The Strengthening the Reporting of Observational Studies in Epidemiology (STROBE) statement: guidelines for reporting observational studies. Lancet 2007;370:1453-1457.

- 35 Moher D, Cook DJ, Eastwood S, Olkin I, Rennie D, Stroup DF: Improving the quality of reports of meta-analyses of randomised controlled trials: the QUOROM statement. Quality of Reporting of Meta-analyses. Lancet 1999;354:1896-1900.

36 Moher D, Liberati A, Tetzlaff J, Altman DG: Preferred reporting items for systematic reviews and meta-analyses: the PRISMA statement. Ann Intern Med 2009;151:264-269, W64.

- 37 Fouque D, Laville M, Haugh M, Boissel JP: Systematic reviews and their roles in promoting evidence-based medicine in renal disease. Nephrol Dial Transplant 1996;11: 2398-2401.

38 Kranke P: Evidence-based practice: how to perform and use systematic reviews for clinical decision-making. Eur J Anaesthesiol 2010;27:763-772.

-39 Normand SL: Meta-analysis: formulating, evaluating, combining, and reporting. Stat Med 1999;18:321-359.

40 Riley RD: Commentary: like it and lump it? Meta-analysis using individual participant data. Int J Epidemiol 2010;39:1359-1361.

-41 Riley RD, Lambert PC, Abo-Zaid G: Metaanalysis of individual participant data: rationale, conduct, and reporting. BMJ 2010; 340:c221.

42 Wardlaw JM, Warlow CP, Counsell C: Systematic review of evidence on thrombolytic therapy for acute ischaemic stroke. Lancet 1997;350:607-614. 
$\checkmark 43$ Counsell CE, Salinas R, Naylor R, Warlow $\mathrm{CP}$ : A systematic review of the randomised trials of carotid patch angioplasty in carotid endarterectomy. Eur J Vasc Endovasc Surg 1997; 13:345-354

44 Juni P, Altman DG, Egger M: Systematic reviews in health care: assessing the quality of controlled clinical trials. BMJ 2001;323:4246.

45 Hallam E, Plaice C: An evaluation of EMBASE within the NHS: findings of the Database Access Project working partnership to extend the knowledge base of healthcare. Health Libr Rev 1999;16:192-203.

46 Egger M, Smith GD: Bias in location and selection of studies. BMJ 1998;316:61-66.

47 Hopewell S, McDonald S, Clarke M, Egger M: Grey literature in meta-analyses of randomized trials of health care interventions. Cochrane Database Syst Rev 2007; MR000010.

48 8.5 The Cochrane Collaboration's tool for assessing risk of bias; in Higgins JPT, Green S (eds): Cochrane Handbook for Systematic Reviews of Interventions. The Cochrane Collaboration, 2008.

49 Hartling L, Ospina M, Liang Y, Dryden DM, Hooton N, Krebs Seida J, et al: Risk of bias versus quality assessment of randomised controlled trials: cross-sectional study. BMJ 2009;339:b4012.

50 DerSimonian R, Laird N: Meta-analysis in clinical trials. Control Clin Trials 1986;7: 177-188.

51 Deeks JJ: Issues in the selection of a summary statistic for meta-analysis of clinical trials with binary outcomes. Stat Med 2002;21: 1575-1600.

52 Higgins JPT, Thompson SG, Deeks JJ, Altman DG: Measuring inconsistency in metaanalyses. BMJ 2003;327:557-560.

53 Thompson SG, Higgins JP: How should meta-regression analyses be undertaken and interpreted? Stat Med 2002;21:1559-1573.

54 Ioannidis JP: Integration of evidence from multiple meta-analyses: a primer on umbrella reviews, treatment networks and multiple treatments meta-analyses. CMAJ 2009;181: 488-493.

55 Cipriani A, Furukawa TA, Salanti G, Geddes JR, Higgins JP, Churchill R, et al: Comparative efficacy and acceptability of 12 newgeneration antidepressants: a multiple-treatments meta-analysis. Lancet 2009;373:746758 .
56 Bucher HC, Guyatt GH, Griffith LE, Walter SD: The results of direct and indirect treatment comparisons in meta-analysis of randomized controlled trials. J Clin Epidemiol 1997;50:683-691.

57 Dias S, Welton NJ, Caldwell DM, Ades AE: Checking consistency in mixed treatment comparison meta-analysis. Stat Med Mar 30; 29:932-944

58 Jadad AR, Moore RA, Carrol D, Jenkinson C, Reynolds JM, Gavaghan DJ, et al: Assessing the quality of reports of randomized clinical trials: is blinding necessary? Controlled Clinical Trials 1996;17:1-12.

59 Berlin JA: Does blinding of readers affect the results of meta-analyses? University of Pennsylvania Meta-analysis Blinding Study Group. Lancet 1997;350:185-186.

60 Kjaergard LL, Villumsen J, Gluud C: Reported methodologic quality and discrepancies between large and small randomized trials in meta-analyses. Ann Intern Med 2001;135 982-989.

61 Pham B, Klassen TP, Lawson ML, Moher D: Language of publication restrictions in systematic reviews gave different results depending on whether the intervention was conventional or complementary. J Clin Epidemiol 2005;58:769-776.

62 Moher D, Pham B, Klassen TP, Schulz KF Berlin JA, Jadad AR, et al: What contributions do languages other than English make on the results of meta-analyses? J Clin Epidemiol 2000;53:964-972.

63 Jefferson T, Rudin M, Brodney Folse S, Davidoff F: Editorial peer review for improving the quality of reports of biomedical studies. Cochrane Database Syst Rev 2007; MR000016.

64 Iansavichene AE, Sampson M, McGowan J Ajiferuke IS: Should systematic reviewers search for randomized, controlled trials published as letters? Ann Intern Med 2008; 148:714-715.

65 McAuley L, Pham B, Tugwell P, Moher D: Does the inclusion of grey literature influence estimates of intervention effectiveness reported in meta-analyses? Lancet 2000;356: $1228-1231$.
66 Buscemi N, Hartling L, Vandermeer B, Tjosvold L, Klassen TP: Single data extraction generated more errors than double data extraction in systematic reviews. J Clin Epidemiol 2006;59:697-703.

67 Horton J, Vandermeer B, Hartling L, Tjosvold L, Klassen TP, Buscemi N: Systematic review data extraction: cross-sectional study showed that experience did not increase accuracy. J Clin Epidemiol 2010;63:289-298.

68 Jadad AR, Cook DJ, Browman GP: A guide to interpreting discordant systematic reviews. CMAJ 1997;156:1411-1416.

69 Goodman SN, Berlin JA: The use of predicted confidence intervals when planning experiments and the misuse of power when interpreting results. Ann Intern Med 1994;121: 200-206.

70 Crumley ET, Wiebe N, Cramer K, Klassen TP, Hartling L: Which resources should be used to identify RCT/CCTs for systematic reviews: a systematic review. BMC Med Res Methodol 2005;5:24.

71 Sampson M, Barrowman NJ, Moher D, Clifford TJ, Platt RW, Morrison A, et al: Can electronic search engines optimize screening of search results in systematic reviews: an empirical study. BMC Med Res Methodol 2006;6:7.

72 Schulze KF, Chalmers I, Hayes RJ, Altman DG: Empirical evidence of bias. JAMA 1995; 273:408-412.

73 Chan AW, Hrobjartsson A, Haahr MT, Gotzsche PC, Altman DG: Empirical evidence for selective reporting of outcomes in randomized trials: comparison of protocols to published articles. JAMA 2004;291:24572465

74 Bassler D, Briel M, Montori VM, Lane M, Glasziou P, Zhou Q, et al: Stopping randomized trials early for benefit and estimation of treatment effects: systematic review and meta-regression analysis. JAMA 2010;303: 1180-1187.

75 Cho MK, Bero LA: The quality of drug studies published in symposium proceedings. Ann Intern Med 1996;124:485-489.

76 Egger M, Davey Smith G, Schneider M, Minder C: Bias in meta-analysis detected by a simple, graphical test. BMJ 1997;315:629634.

77 Ioannidis JP: Effectiveness of antidepressants: an evidence myth constructed from a thousand randomized trials? Philos Ethics Humanit Med 2008;3:14. 


\section{Editorial Comment}

Meguid El Nahas, Sheffield

Bello and his colleagues in Edmonton present an expert review on the validity of systematic reviews and meta-analyses. They make the very important point that many of the perceived limitations of meta-analysis are not inherent in the methodology, but actually represent deficits in the conduct or reporting of individual primary studies. Unfortunately, all too often, nephrologists draw conclusions and practice guidelines from meta-analyses without full appreciation of the quality and/or the heterogeneity of the studies included.
Until recently, most clinical trials in nephrology were too small to be conclusive; mixing them all up into a meta-analysis seemed to be the misleading magic formula; we have seen such practices with interventions ranging from dietary protein restriction to ACE inhibition in CKD! A better understanding of the application and limitations of meta-analyses is provided in this review that may prevent in the future inappropriate conclusions based on such data manipulation. 